Toward the Automated Synthesis of Cooperative Mobile Robot Teams ${ }^{*}$

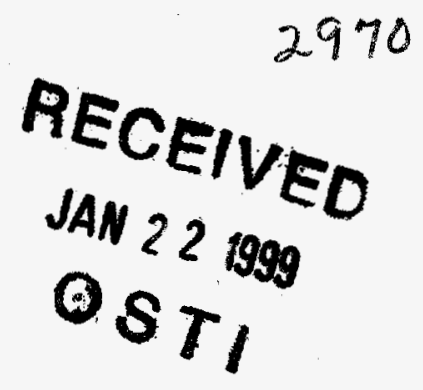

\author{
Lynne E. Parker \\ Center for Engineering Science Advanced Research \\ Computer Science and Mathematics Division
}

\author{
Oak Ridge National Laboratory
}

\begin{abstract}
The submitted manuscript has been authored by a contractor of the U.S. Government under contract No. DE-AC05-96OR22464. Accordingly, the U.S. Government retains a nonexclusive, royalty -free license to publish or reproduce the published form of this contribution, or allow others to do so, for U.S. Government purposes.
\end{abstract}

Submitted to SPIE Mobile Robots XIII

Boston, MA

November 1998

\footnotetext{
* Research sponsored by the Engineering Research Program of the Office of Basic Energy Sciences, U.S. Department of Energy, under Contract No. DE-AC05-960R22464 with Lockheed Martin Energy Research Corporation.
} 


\section{DISCLAIMER}

This report was prepared as an account of work sponsored by an agency of the United States Government. Neither the United States Government nor any agency thereof, nor any of their employees, make any warranty, express or implied, or assumes any legal liability or responsibility for the accuracy, completeness, or usefulness of any information, apparatus, product, or process disclosed, or represents that its use would not infringe privately owned rights. Reference herein to any specific commercial product, process, or service by trade name, trademark, manufacturer, or otherwise does not necessarily constitute or imply its endorsement, recommendation, or favoring by the United States Government or any agency thereof. The views and opinions of authors expressed herein do not necessarily state or reflect those of the United States Government or any agency thereof. 


\section{DISCLAIMER}

Portions of this document may be illegible in electronic image products. Images are produced from the best available original document. 


\title{
Toward the automated synthesis of cooperative mobile robot teams
}

\author{
Lynne E. Parker \\ Center for Engineering Sciences Advanced Research \\ Computer Science and Mathematics Division \\ Oak Ridge National Laboratory, Oak Ridge, TN 37831-6355 USA \\ Email: ParkerLE@ornl.gov
}

\begin{abstract}
A current limitation in the real-world use of cooperating mobile robots is the difficulty in determining the proper team composition for a given robotic application. Present technology restricts the design and implementation of cooperative robot teams to the expertise of a robotics researcher, who has to develop robot teams on an applicationspecific basis. The objective of our research is to reduce the complexity of cooperative robotic systems through the development of a methodology that enables the automated synthesis of cooperative robot teams.

We propose an approach to this problem that uses a combination of the theories of sensori-computational systems and information invariants, building on the earlier work of Donald, Rus, et al. We describe the notion of defining equivalence classes that serve as fundamental building blocks of more complex cooperative mobile robot behaviors. We postulate a methodology for framing mission requirements in terms of the goals and constraints of the problem, incorporating issues such as multi-robot interference, communication, control strategy, robot complexity, and so forth, into the mechanism. Our initial work restricts the robot application and design space to three multi-robot application domains we have previously studied and implemented: keeping formation, "mock" hazardous waste cleanup, and cooperative observation. This paper presents the foundational ideas upon which our approach to cooperative team design is based.
\end{abstract}

Keywords: Cooperative behaviors, behavior synthesis, multi-robot learning

\section{INTRODUCTION}

As the use of cooperative mobile robotics moves from theoretical and laboratory studies towards use in real-world applications, a methodology is needed to determine the proper robot team composition for a given mission. The question to be answered is: given a pool of heterogeneous robots and a mission to be accomplished, what is the proper composition of robots for the team, and what strategy of cooperation and interaction should they use?

In recent years, the interest in cooperative robotic systems has grown significantly. A primary reason for this growing interest is the recognition of the large number of application domains in which cooperative robotic systems can be used, including the following: (1) military applications, such as surveillance, reconnaissance, and demining, (2) industrial applications, such as cleaning and earthmoving, and (3) space exploration applications, such as rock gathering and searching for water. Advantages that have been recognized for cooperative teams include increased robustness through redundancy, a potential for decreased mission completion time through parallelism, and a possibility for decreased individual robot complexity through heterogeneous robot teams.

A great deal of research has focused on the development of cooperative control strategies that enable teams of robots to work together in these applications. Much success is beginning to be achieved in this area, with the development of several control architectures and approaches for cooperative teams. The field has gained a basic understanding of many of the important issues in cooperative teams, including swarm versus intentional cooperation, implicit cooperation versus robot awareness, implicit versus explicit communication, increased coverage versus interference, and local versus global cooperative control.

However, it would greatly speed the widespread practical application of multi-robot systems if a methodology were available that could enable a system designer to automatically generate cooperative robot solutions for the specific application at hand. At present, the field is a long way from being able to accomplish cooperative team design. Little previous research has addressed this topic. Most previous research related to this topic involves 


\section{Most Commonly Used Approach for \\ Cooperative Team Design}

Mission Specifications
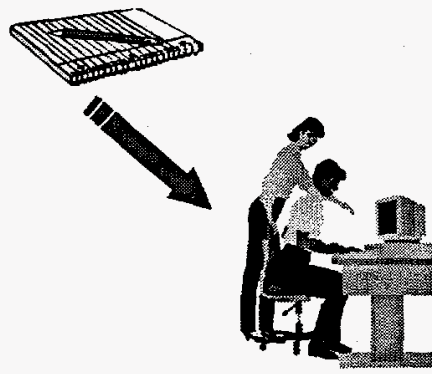

Robot team for specific mission

Researchers build

\& program robots
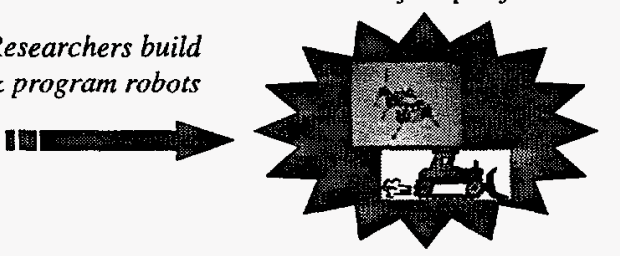

Figure 1. The most common approach to cooperative team design is to custom-build a specific robot team for a specific mission.

the evolutionary design of cooperative behaviors for simple simulated agents in simple worlds. Floreano ${ }^{1}$ uses genetic algorithms to evolve obstacle avoiding and light-homing behaviors for a small robot. Cliff $e t$ al. ${ }^{2}$ use genetic algorithms to create navigation strategies that use simple (one-pixel) vision. Sims ${ }^{3}$ uses competitive co-evolution to create simulated creatures that have to share a common resource. Hillis ${ }^{4}$ also uses co-evolution, in this case to evolve sorting algorithms that operate on various types of data sets. Little previous work addresses the design of teams of more complex cooperative agents.

This paper explores this issue, examining the need for cooperative team design and a proposed approach toward this end. The next section compares the desired capabilities for cooperative team design with the current approaches most commonly employed. We then, in the following sections, outline a proposed approach toward the goal of automated cooperative team design that we are developing. Section 5 examines three previously-implemented cooperative applications that we are using as case studies in the development of our approach to cooperative team design. We conclude in section 6 with a description of our ongoing and future work in this area.

\section{THE NEED FOR COOPERATIVE TEAM DESIGN}

The most commonly used approach to cooperative team design is illustrated in Figure 1 . In this approach, the robotics researcher considers a specific mission to be solved using a cooperative robotics approach. He or she then either designs specific robots for that mission or uses the robots available in the laboratory to build the cooperative software specific to that mission. In either case, the resulting implementation usually provides little guidance to assist in the design of the next cooperative team for another application. Instead, the design is limited because it is mission-specific.

More recent research in cooperative robotics enables a more generic approach to cooperative robot team design. Illustrated in Figure 2, this approach allows researchers to build a pool of heterogeneous robots that can perform a variety of applications within certain domains. At a later time, the (human) system designer can bring together the desired robots to solve a specific mission that arises. The ALLIANCE ${ }^{5,6}$ architecture was designed to enable this more generic approach to cooperative robotics, providing a mechanism that enables teams of robots to work together without advanced knowledge of the robot team composition. By programming each robot in advance using the ALLIANCE approach, we can know that the robots will work together properly when applied to a mission at some future time.

However, even this more generic approach does not provide guidance to the questions: Given a specific mission, what is the "optimal" cooperative mobile robot team composition? How do you design a multi-robot team for a specific mission that "optimizes" the issues of cost, fault tolerance, efficiency, interference, individual robot complexity, team size, and so forth? Figure 3 illustrates this ultimate goal of automated team design. In this context, the definition of "optimality" will vary, depending upon the mission. The optimality criteria is part of the mission specifications. 


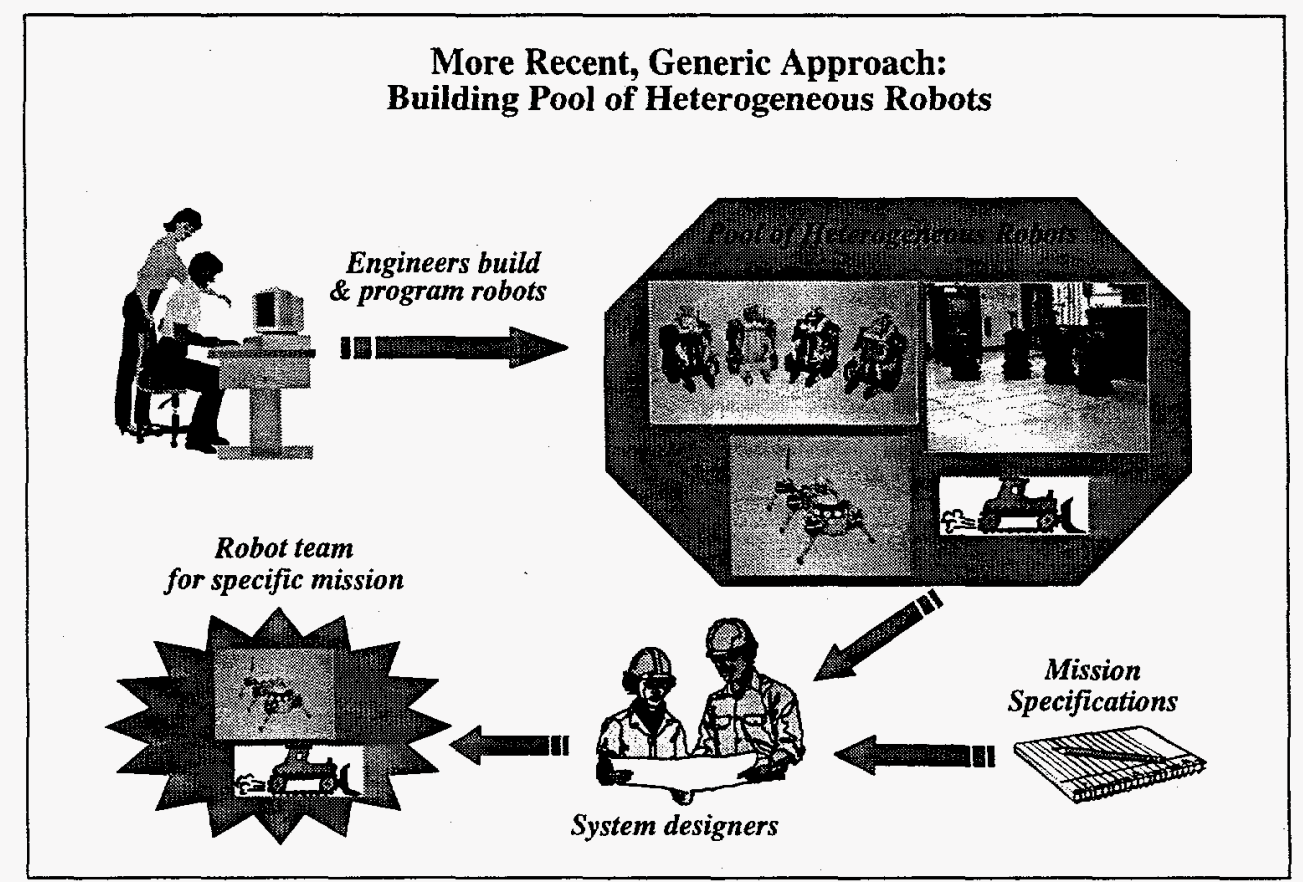

Figure 2. In more recent approaches, a robot pool can be generated in advance, and applied to specific applications when they arise. More generic approaches, such as ALLIANCE, enable the robot teams to be composed in advance of their use in a specific application.

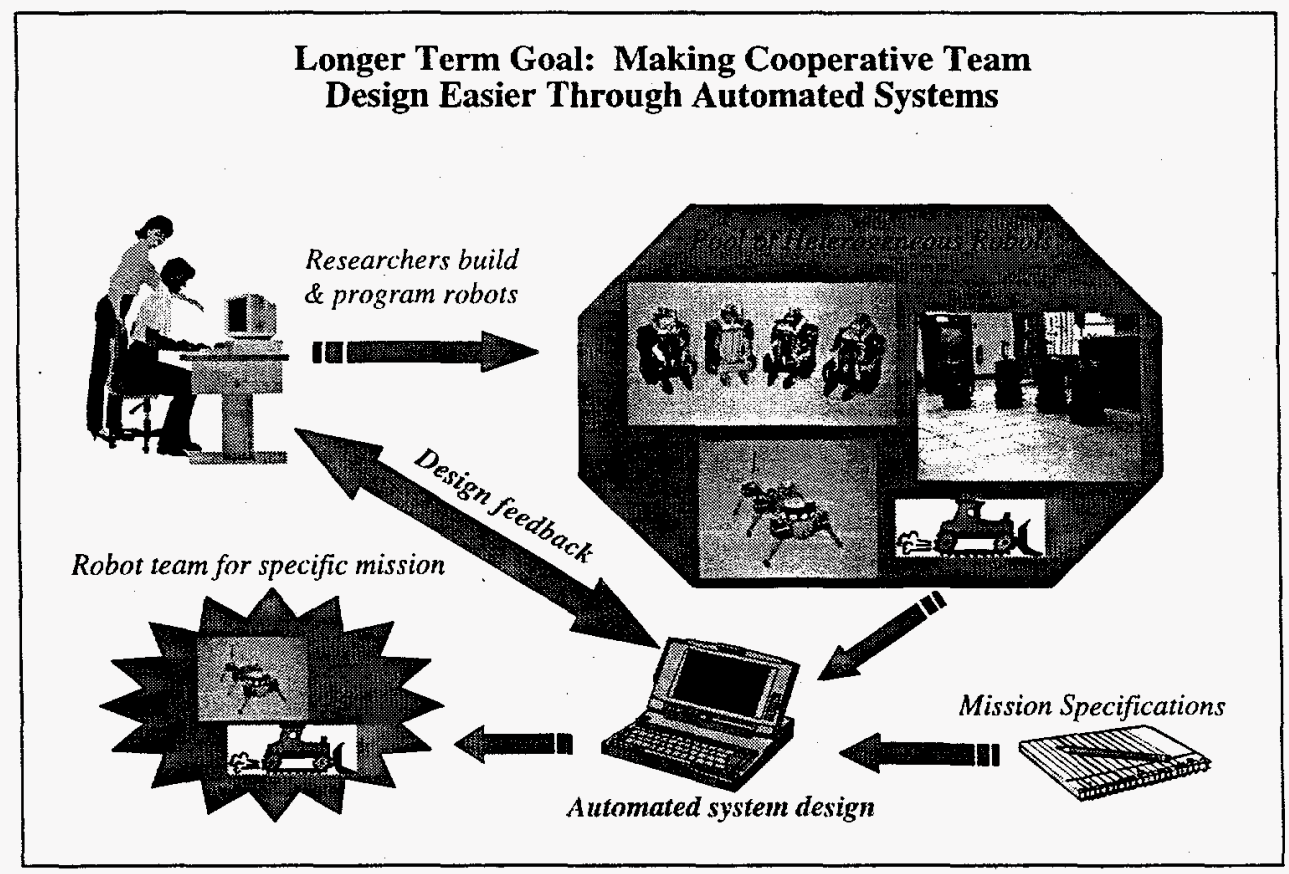

Figure 3. The ultimate goal in automated team design is to be able to automatically compose the proper team for a specific mission. Having this capability will also provide insight and feedback into the proper design of the individual robot as part of the heterogeneous robot pool. 


\section{REQUIREMENTS FOR AUTOMATED TEAM DESIGN}

A number of issues must be addressed before a methodology can be developed for automated team design. Most of these issues involve developing descriptions of components of cooperative multi-robot teams and their interactions for use in a rigorous approach to automated design. In our work, we have identified those issues of importance toward this goal, but have only begun to develop the rigorous descriptions required to quantify each issue. In this section, we make note of the many complex issues that must be incorporated if an automated design solution is to be successful.

First of all, a mechanism, or "language", is needed to describe the specifications of the mission of interest. This language must be broad enough to be appropriate for large classes of application domains. Along with the mission specifications are the metrics to be used to measure the performance of the mission. Ideally, these metrics will include both components that are independent of a specific robot team (such as time), and components that are dependent upon the given robot team (such as energy expenditure). An approach for team design also requires a method of describing individual robots - including both physical capabilities and individual behaviors. Beyond the individual robot descriptions are needed the descriptions of the interactions of robot team members, as well as the costs of individual subsystems (such as the use of certain sensors) and interactive components (such as communication).

An approach to cooperative team design must also take into account a number of other issues. These issues include fault tolerance, which incorporates the probability of robot failure; efficiency, which balances the trade-off between more robots versus increased energy usage; interference, which balances the tradeoff between more robots versus an increased generation of conflicts; individual robot complexity, which finds a balance between homogeneous versus heterogeneous robots, and simple versus complex individual robot design; and local versus global control, which involves decisions on issues of interaction through local information versus more global coordination, perhaps through communication.

Clearly, with all of the above issues impacting cooperative teams, the goal of automated tcam design is very ambitious and challenging. While we are far from having a complete solution to this goal, we have identified an approach that we believe has high potential towards this end. The following section describes the basis upon which we propose to build a methodology for automated cooperative team design.

\section{PROPOSED APPROACH TO AUTOMATED TEAM DESIGN}

Our proposed approach to automated team design combines the theories of sensori-computational systems and information invariants, building upon the earlier work of Donald et al. ${ }^{7}$ We expect that the use of these theories should enable the creation of automated cooperative behavior solutions that are re-usable within an application domain through reinstantiation with modules from equivalence classes of components. In this design, a sensoricomputational system (SCS) is a module that computes a function of its inputs and its current pose or position. ${ }^{7}$ For example, a wall-following sensori-computational system could compute the velocity and steering commands required to move a robot along a wall, based upon the values of the robot's range sensors. Since a wall-following sensori-computational system (for example) can be designed in many different ways, one can define a mapping from tasks or sensors to equivalence classes of sensori-computational systems that require the same intrinsic information. This intrinsic information is termed an information invariant, which measures the complexity of the information required to perform a task. In earlier work, Donald ${ }^{7}$ showed that reductions from one sensori-computational system to another can occur based upon equivalences between communication, internal state, external state, computation, and sensors. The significance of the reductions is that they guarantee that one sensory system can be built from another without adding information complexity.

We propose to use this notion of information invariants to define equivalence classes of sensori-computational systems that serve as fundamental building blocks (or basis functions) of more complex cooperative robotic behaviors. Optimization techniques will then be used to generate cooperative robot teams that best fit the specifications of the desired mission.

More specifically, the overall approach is as follows. Given a mission, first determine its information invariants. Then, the information invariants are mapped to equivalence classes of robot teams that can solve the mission. The robot team equivalence classes define components of the robot team that are required for that mission, in terms of sensors, behaviors, actuators, communication, and cooperative control. Then, the minimal set of robot team components is selected, based upon component costs. The mission metrics are then used as optimization criteria to distribute the collective set of team components across individual robots that will compose the team. 


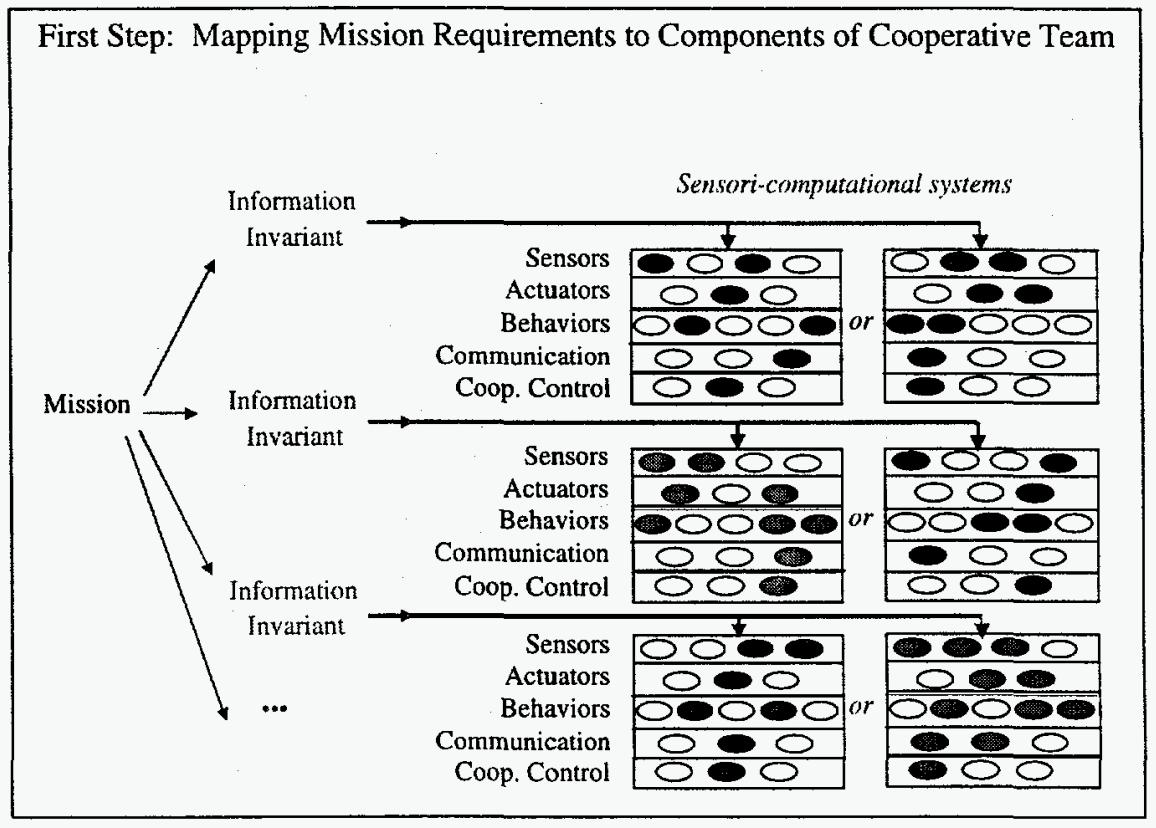

Figure 4. The first step of the approach involves defining the information invariants required by the mission, and then mapping these invariants to the equivalence class of sensori-computational systems that can achieve that invariant.

Figures 4 through 6 illustrate this process. As shown in Figure 4, the mission is mapped to information invariants, which are mapped to a number of possible sensori-computational systems that can achieve each information invariant. The ovals in the diagram refer to alternative components that can be used in the design. The shaded ovals represent those components selected for a particular design. Any single information invariant can potentially be achieved by a number of different sensori-computational systems. The figures shows two such SCSs per each information invariant, but an actual application may have more.

The next step, as illustrated in Figure 5, requires selecting the most efficient team components that achieve the information invariants, optimized based upon the predetermined subsystem costs. Shown in the diagram is a simple cost function that weights each module equivalently. Thus, an optimization function based upon a minimal cost would select the combination of sensori-computational elements that minimizes the total number of modules. The minimal such combination is shown in Figure 5 for the example illustrated.

As shown in Figure 6, the final step of the approach requires mapping the required cooperative team components to individual robot team members, based upon the defined metrics of the mission. The result of the previous step indicates the required components of the robot team as a whole, but it does not indicate how those components should be distributed to the individual robots. Robot team size and capabilities of individual robots are determined at this step.

\section{CASE STUDIES TO BOUND INITIAL DEVELOPMENT}

To ground these ideas in specific applications, our approach to developing a methodology for automated team design is to restrict the mission design space and the robot design space to case studies of cooperative mobile robot systems that we have previously implemented. Initially, we are working with three such case studies: keeping formation, "mock" hazardous waste cleanup, and cooperative observation of multiple moving targets. After the approach for automated team design has been fully studied with these three case studies, we will then generalize the approach to a broader application domain. At that point, we may include additional cooperative robot applications that we have implemented, such as the cooperative box pushing application described in. ${ }^{8}$

Thus far, we are just beginning the analysis of these case studies for the purpose of automated team design. For each of these three case studies, we have identified the mission metric, the information invariants, and a qualitative notion of information costs. Ongoing work is aimed at mapping these information requirements to the equivalence 


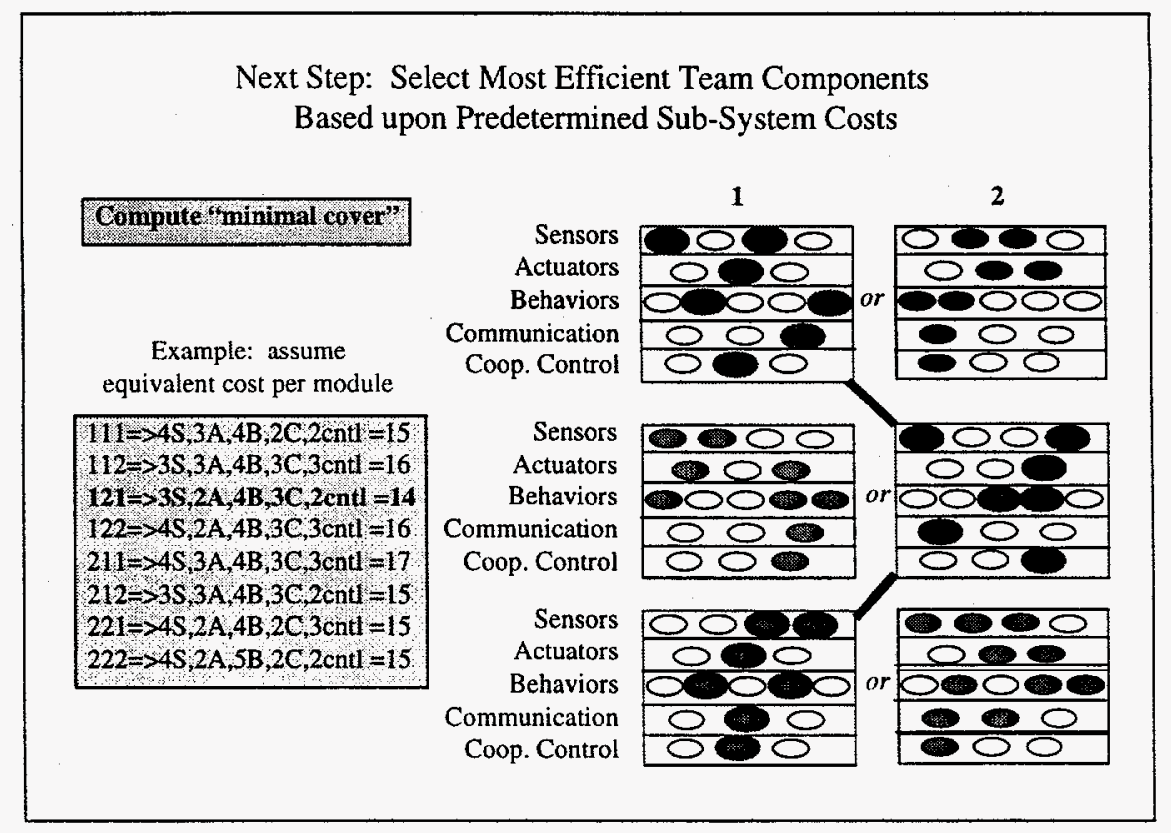

Figure 5. The next step of the approach involves selecting the subset of sensori-computational systems that is the most efficient solution, based upon predetermined subsystem costs.

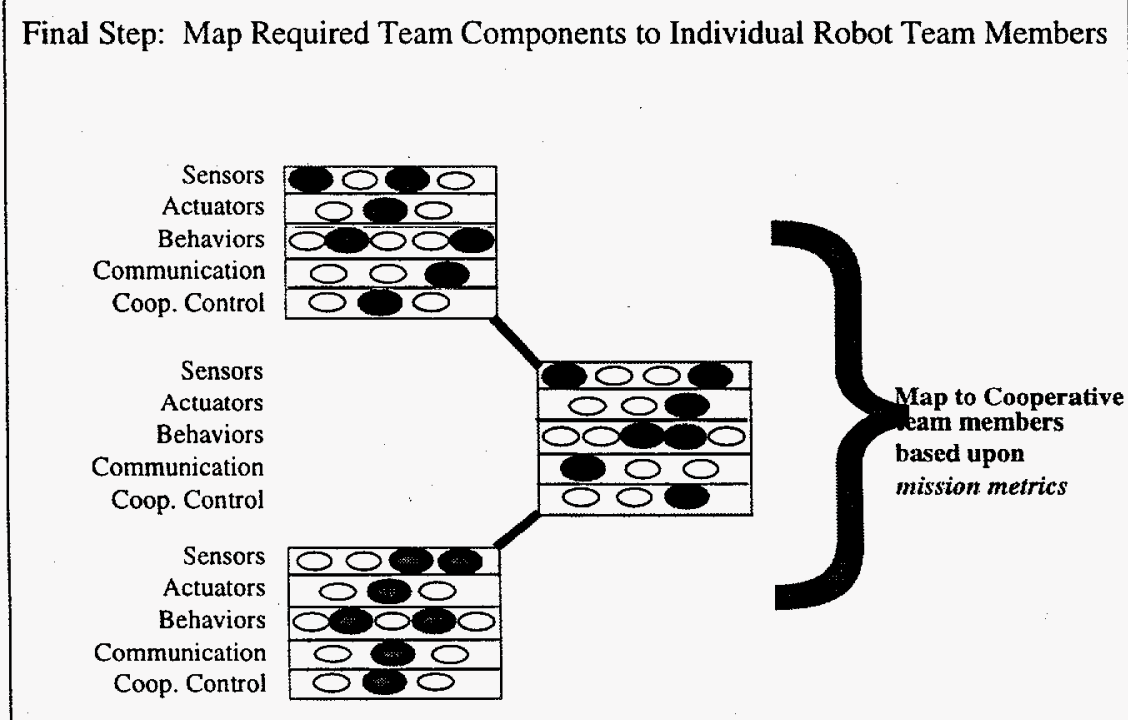

Figure 6. The final step of the approach involves distributing the required team components to individual robot team members, based upon the mission metrics. 


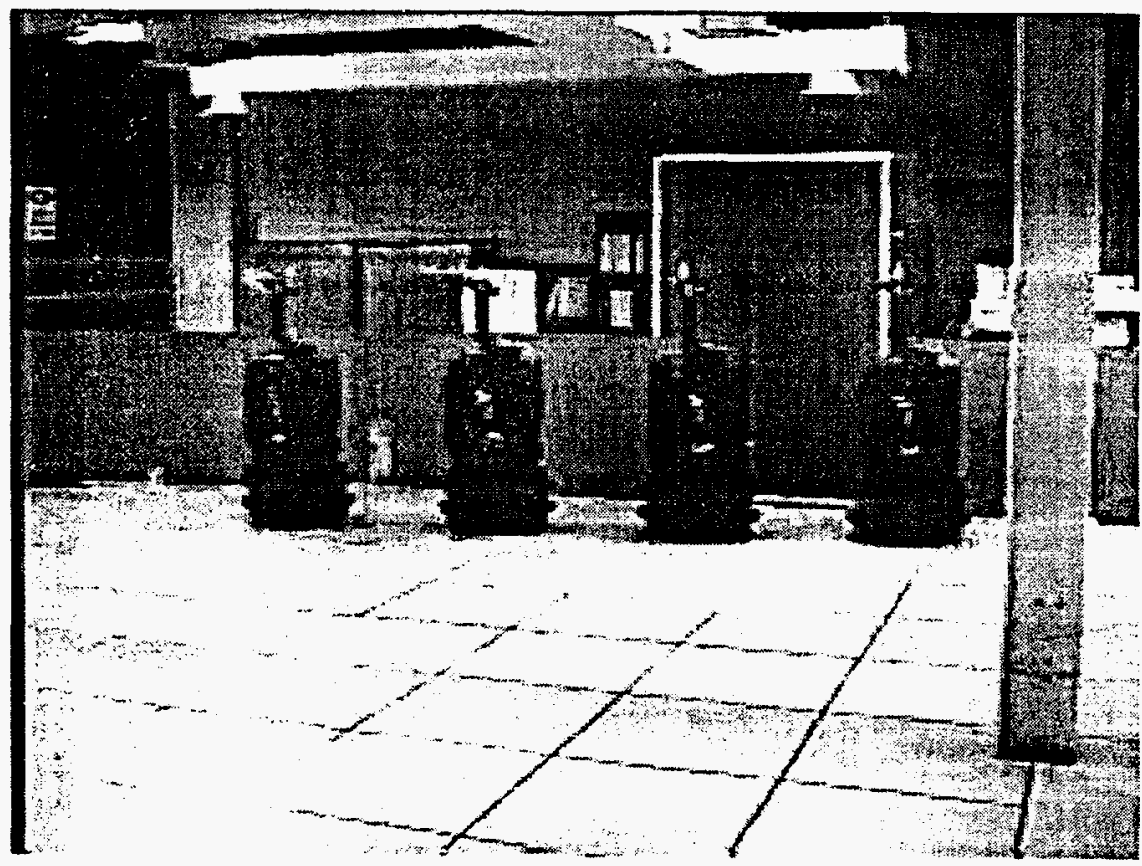

Figure 7. Four robots moving in column (front-to-back) formation.

classes of sensori-computational systems required to achieve these information invariants. The following subsections describe these case studies

\subsection{Keeping formation}

In many cooperative robotic applications, it is necessary for robot team to move in an orderly fashion from one location to another. We have written algorithms that cnable robot team members to maintain two types of formations: column and line. Column formation requires robots to maintain formation front-to-back. In this implementation, the first robot performs wall following, while the other robots follow each other in formation using range or vision sensing. The photos in Figure 7 show the robot team moving in column formation. Figure 8 slows a simulated set of robots moving in line formation. In line formation, the robots are recpired to maintain a side-by-side configuration. In all of these types of formations, robots attempt to manitain a desired position relative either to nearby robots, or to a predefined robot team leader. Refer to ${ }^{2,5}$ for studies on the tradeoffs in local versus global control for cooperative formations.

In this application, the mission metric is defined in terms of a normalized cumulative formation crror. This is the accumulated distance from the desired formation position of each robot, measured at discrete time increments for the duration of the mission. More specifically, at a given time $t$, the formation error, $f e_{t}$, is given by

$$
f c_{t}=\sum_{i \neq l e a d c r} d_{i}
$$

where $d_{i}$ is the distance between the curent position of robot $i$ and the proper formation position of robot $i$, based on the formation leader's current location. The mission metric is clefined as the cumulative formation crror, cum_ $f$ e, which is defined as:

$$
u r n_{-} f e=\sum_{t=0}^{t_{\max }} f e_{t}
$$

The information invariants are different for leader robots versus follower robots. For leader robots, the information invariants are defined as: 

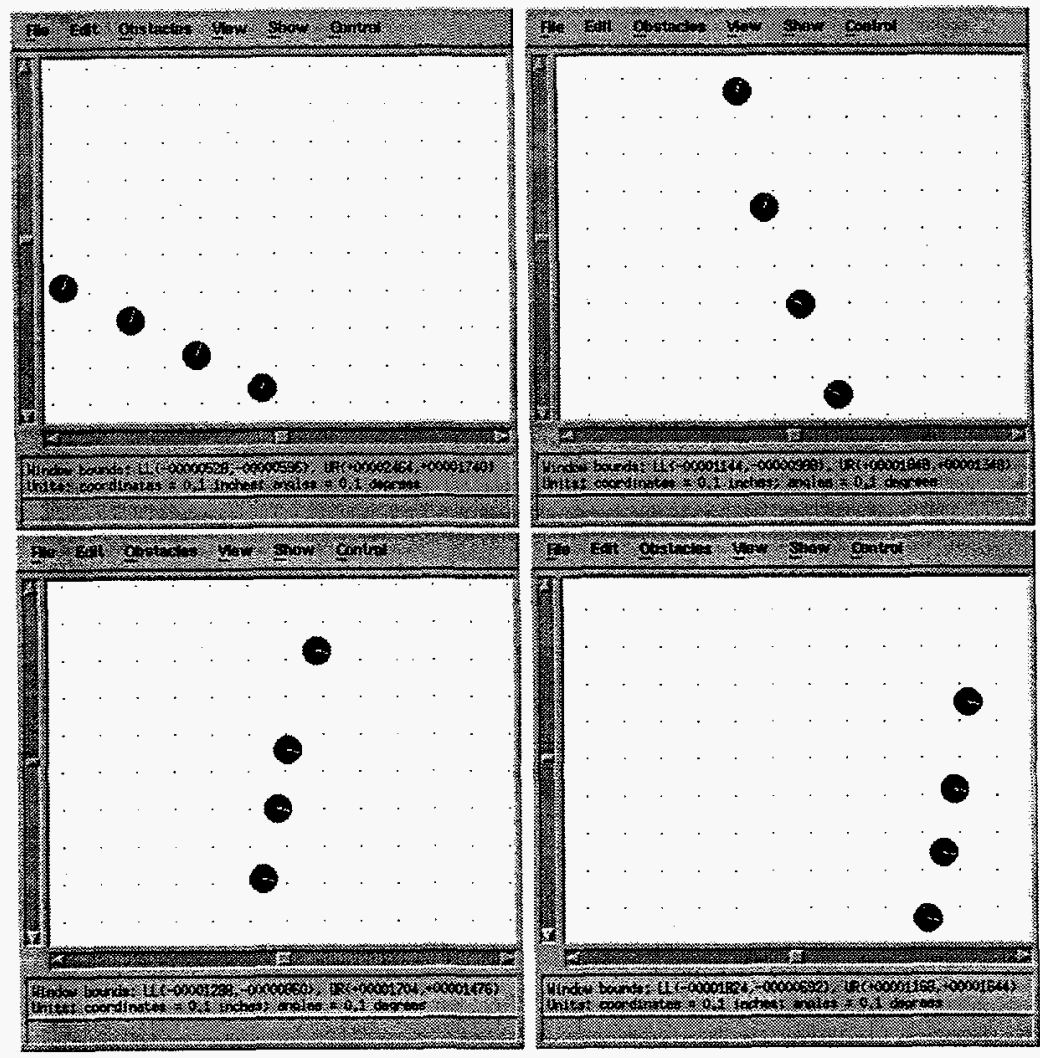

Figure 8. Simulated robots moving in line (side-by-side) formation.

- Current location

- Current goal location

- Location of neighbor robots

For follower robots, the information required to perform the task is different based upon the control strategy used. We do not go into detail here on the various control strategies; they are reported in. ${ }^{9}$ Instead, we simply list the information we have identified that is required to execute each of the control strategies:

- Strategy I:

- Current location

- Location of neighbors

- Strategy II:

- Current location

- Location of neighbors

- Location of leader

- Strategy III:

- Current location

- Location of neighbors

- Current goal location 


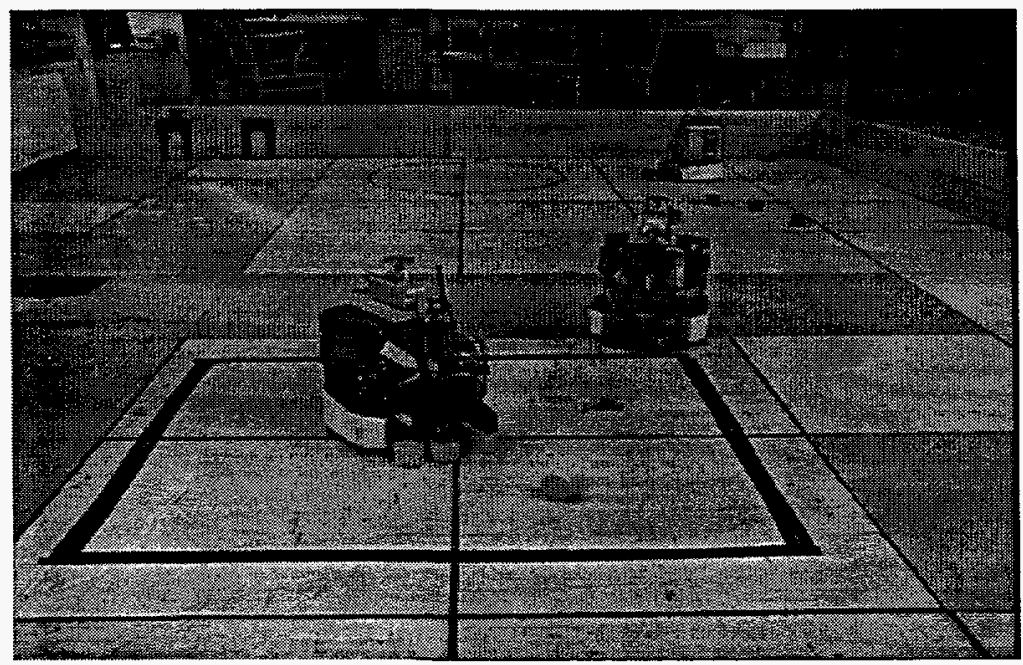

Figure 9. A robot team performs the "mock" hazardous waste cleanup application.

- Strategy IV:

- Current location

- Location of neighbors

- Current goal location

- Next goal location

The costs associated with obtaining this information are identified qualitatively as follows:

- Location of neighbors: Per unit time, range or vision processing for neighbor recognition and position estimation, or GPS communication

- Location of leader: Per unit time, range or vision processing for neighbor recognition and position estimation, or GPS communication

- Current goal location: Periodic communication

- Next goal location: Periodic communication

- Current position: Internal navigation system (INS) localization processing or GPS communication

\section{2. "Mock" hazardous waste cleanup}

The "mock" hazardous waste cleanup application requires two artificially "hazardous" waste spills in an enclosed room to be cleaned up by a team of three robots. This mission requires robot team members to locate the two waste spills, move the two spills to a goal location, while also periodically reporting the team progress to humans monitoring the system. Figure 9 shows the robots while they are performing this application. Refer to ${ }^{6}$ for more details on this implementation.

In this application, the mission metric is defined in three parts - one for each subtask of the mission. Specifically, the metrics are as follows:

For the find location task, the metric is to minimize the collective time or energy spent locating pucks:

$$
\min \sum_{i=1}^{n} \sum_{t=0}^{t_{m a x}}\left(a\left(r_{i}, t\right)=\text { find_locations }\right)
$$

where $a\left(r_{i}, t\right)$ returns the task that robot $r_{i}$ is performing at time $t$. 
For the move spills task, the metric is to maximize the number of pucks moved per collective time:

$$
\max \sum_{i=1}^{n} \sum_{t=0}^{t_{\max }}\left(a\left(r_{i}, t\right)=\text { move_spill }\right)
$$

For the report progress task, the metric is to minimize the difference between the actual rate of progress reports and the desired rate:

$$
\min \left|\frac{\text { actual(prog_rpt_rate })}{\Delta t}-\frac{\text { desired(prog_rpt_rate })}{\Delta t}\right|
$$

The information required to perform these tasks is as follows:

- Find location:

- Current location

- History of perviously searched locations (can be implicit in the control approach)

- Move pucks:

- Current location

- Location of pucks

- Puck detection

- Goal location

- Report progress:

- Current location

- Progress report location

- Desired rate of progress reports

- Time since last progress report

The qualitative cost associated with obtaining this information is as follows:

- Current location: INS localization processing or GPS communication

- History of previously searched region: Time to explore environment

- Location of puck region: One-time communication

- Puck detection: Per unit time, range processing

- Goal location: One-time communication

- Progress report location: One-time communication

- Desired rate of progress reports: One-time communication

- Time since last progress report: Stored time, or periodic communication 

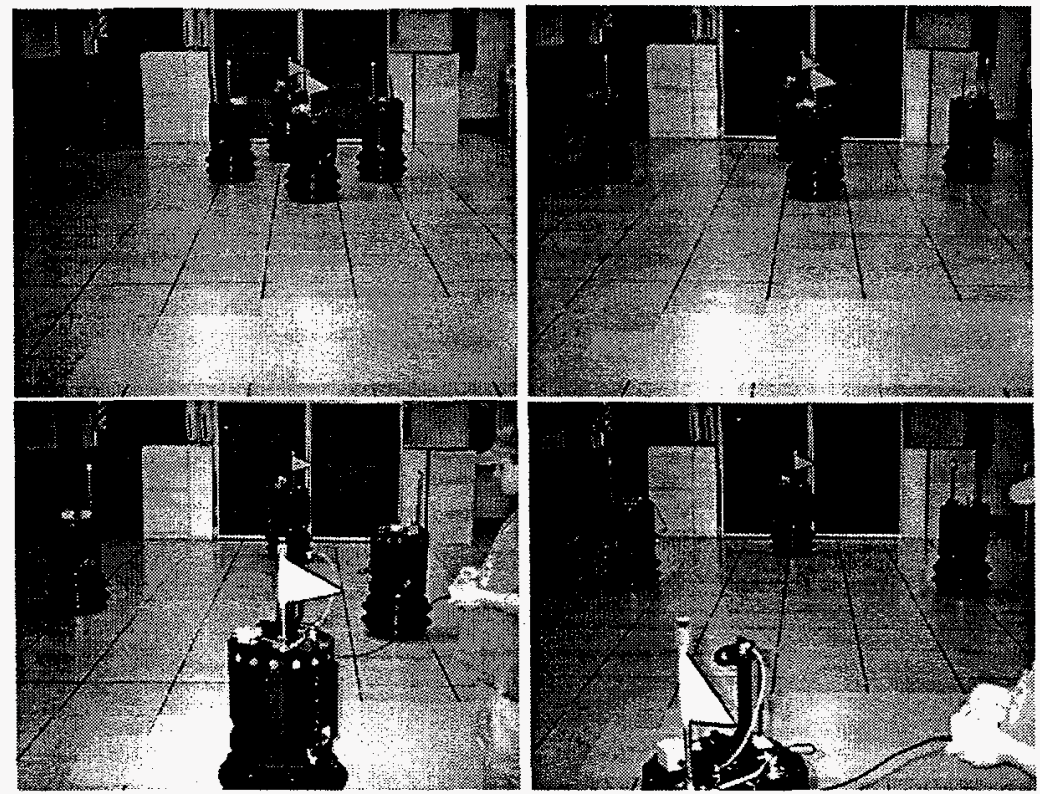

Figure 10. These photos show a team of robots performing the cooperative observation task.

\subsection{Cooperative multi-robot observation of multiple moving targets}

In this application, a team of robots is required to observe the movements of targets moving through a specified area of interest. Because the sensor ranges of the robots and the size of the robot team are limited, fixed robot positions are not sufficient to maintain the entire area of interest under observation. Thus, the robot team members must move dynamically over time based upon the movements of the targets and the other robots on the team to ensure that as many targets as possible remain under observation. In previous work, ${ }^{10,11}$ we have developed a distributed approximate approach to solving this problem that combines low-level multi-robot control with higher-level control. The low-level control is described in terms of force fields emanating from the targets and the robots. The higher level control is presented in the ALLIANCE formalism, ${ }^{6}$ which provides mechanisms for fault tolerant cooperative control, and allows robot team members to adjust their low-level actions based upon the actions of their teammates. We have implemented and compared four different control approaches to cooperative observation: (1) FIXED, where robots maintain fixed positions uniformly distributed over the area, (2) RANDOM, where robots move randomly, (3) LOCAL, which uses local force vectors, without any higher-level control, and (4) A-CMOMMT, which combines low-level and higher-level control. Figure 10 shows an implementation of this work on team of robots.

The mission metric defined for this application is to maximize the average number of targets under observation during the mission:

$$
\sum_{t=0}^{t_{\max }} \sum_{j=1}^{n} \bigvee_{i=1}^{m} a_{i j}(t)
$$

where $a_{i j}(t)=1$ if target $j$ is under observation by robot $i$ at time $(t)$, and 0 otherwise.

The information invariants defined for this task are as follows:

- Location of local targets

- Location of local robots

The qualitative cost of obtaining this information is defined as:

- Location of local targets: Per unit time, vision or range processing for target localization, or GPS communication

- Location of local robots: Per unit time, vision or range processing for robot localization, or GPS communication 


\section{SUMMARY AND FUTURE WORK}

In this paper, we have outlined a proposed approach towards the ultimate goal of automated cooperative team design. This approach involves first defining the information invariants of a given mission, and then mapping those information invariants to equivalence classes of sensori-computational systems that can achieve those invariants. An optimal subset of sensori-computational systems is then selected based upon the costs of the individual subsystems. This subset defines the collection of modules that must be present in the cooperative team as a whole to achieve the mission. The final step involves distributing these components to the individual robot team members, based upon the mission metrics defined in the original mission specifications.

We are beginning our study of this approach by narrowing the mission and robot design space to three cooperative robot case studies that we have previously implemented. For these three case studies, we have defined the mission metrics, information invariants, and information costs associated with achieving the mission goals. Our ongoing work now involves mapping the information invariants for these case studies to the equivalence classes of sensoricomputational systems required to achieve these invariants. We will then examine the optimization issues needed to select a minimal subset of components, and for mapping these components to individual robot team members.

The aim of this research in cooperative team design is to develop a methodological approach that can lead to an automated solution to the difficult question: How do you design cooperative mobile robot teams for specific missions to optimize the issues of: cost, fault tolerance, efficiency, interference, individual robot complexity, cooperative control, and team size? Clearly, much work remains to be done to reach this ambitious goal. But if successful, we believe that an automated solution for cooperative team design will have a significant impact toward the widespread use of cooperative robot teams in real-world applications.

\section{ACKNOWLEDGMENTS}

This research is sponsored by the Office of Engineering Research, Basic Energy Sciences of the U.S. Department of Energy, under contract No. DE-AC05-96OR22464 with Lockheed Martin Energy Research Corporation.

\section{REFERENCES}

1. D. Floreano, "Patterns of interactions in shared environments," in Proceedings of the First European Conference on Artificial Life, pp. 347-366, 1993.

2. D. T. Cliff, P. Husbands, and I. Harvey, "Evolving visually guided robots," in Proceedings of the Second International Conference on Simulation of Adaptive Behavior SAB92, pp. 374-383, 1992.

3. K. Sims, "Evolving 3D morphology and behavior by competition," in Proceedings of ALIFE IV, pp. 28-39, 1994.

4. W. D. Hillis, "Co-evolving parasites improve simulated evolution as an optimization procedure," Physica 42, pp. 228-234, 1990.

5. L. E. Parker, Heterogeneous Multi-Robot Cooperation. $\mathrm{PhD}$ thesis, Massachusetts Institute of Technology, Artificial Intelligence Laboratory, Cambridge, MA, February 1994. MIT-AI-TR 1465 (1994).

6. L. E. Parker, "ALLIANCE: An architecture for fault-tolerant multi-robot cooperation," IEEE Transactions on Robotics and Automation 14(2), pp. 220-240, 1998.

7. B. R. Donald, J. Jennings, and D. Rus, "Towards a theory of information invariants for cooperating autonomous mobile robots," in Proceedings of the International Symposium of Robotics Research, (Hidden Valley, PA), October 1993.

8. L. E. Parker, "ALLIANCE: An architecture for fault tolerant, cooperative control of heterogeneous mobile robots," in Proc. of the 1994 IEEE/RSJ/GI Int'l Conf. on Intelligent Robots and Systems (IROS '94), pp. 776783, (Munich, Germany), Sept. 1994.

9. L. E. Parker, "Designing control laws for cooperative agent teams," in Proceedings of the IEEE Robotics and Automation Conference, pp. 582-587, (Atlanta, GA), 1993.

10. L. E. Parker, "Cooperative motion control for multi-target observation," in Proceedings of the 1997 IEEE/RSJ International Conference on Intelligent Robots and Systems (IROS '97), pp. 1591-1598, 1997.

11. L. E. Parker, "Distributed algorithms for multi-robot observation of multiple moving targets," accepted for publication in Autonomous Robots, 1999. 